\title{
EXTRAINTESTINAL MANIFESTATIONS AND INTESTINAL COMPLICATIONS IN PATIENTS WITH CROHN'S DISEASE: ASSOCIATIONS WITH SOME CLINICO-LABORATORY FINDINGS, IMMUNOLOGICAL MARKERS AND THERAPY
}

\author{
Ts. Velikova ${ }^{1}$, R. Miladinova ${ }^{2}$, E. Ivanova-Todorova ${ }^{3}$, D. Kyurkchiev ${ }^{3}$, Z. Spasova ${ }^{4}$ \\ ${ }^{1}$ Clinical Immunology, University Hospital "Lozenetz" - Sofia, Bulgaria \\ ${ }^{2}$ Clinic of Nephrology, University Hospital "Sv. Ivan Rilski”, Medical University - Sofia, Bulgaria \\ ${ }^{3}$ Laboratory of Clinical Immunology, University Hospital "Sv. Ivan Rilski", \\ Department of Clinical Laboratory and Clinical Immunology, Medical University - Sofia, Bulgaria \\ ${ }^{4}$ Clinic of Gastroenterology, University Hospital "Sv. Ivan Rilski”, Medical University - Sofia, Bulgaria
}

\begin{abstract}
Crohn's disease (CD) may have some severe complications that pose an increasing health burden and negatively impact the quality of life. There are two major types - intestinal and extraintestinal complications, in which immune and non-immune mechanisms take place. We aimed to search for some associations between specific extraintestinal manifestation and intestinal complications in $C D$ patients with some clinicallaboratory findings, immunological markers, and the therapy administered. We examined retrospectively medical files of 26 patients with $C D$ at mean age $42 \pm 13$ years, including the laboratory results. The immunological markers fecal calprotectin (FC) and fecal lactoferrin (FL) were assessed in frozen fecal samples of the chosen patients. Seventy-three percent of the investigated $C D$ patients had some extraintestinal manifestation and/or intestinal complications, at least 13/26 had intestinal complications. All three patients with extraintestinal signs were positive for $F C$ and $2 / 3$ were positive for $F L$. We observed a higher serum level of CRP (24.49 mg/l vs. $3.13 \mathrm{mg} / \mathrm{l}, p=0.010)$, slightly lowered serum level of hemoglobin (120 g/l vs. $145 \mathrm{~g} / \mathrm{l}, p=0.044)$ and about 2-fold lower iron level $(7.23$ $\mu \mathrm{mol} / \mathrm{I}$ vs. $14.0 \mu \mathrm{mol} / \mathrm{l}, p=0.019)$ in patients with intestinal complications compared to patients without complications, respectively. Four out of thirteen patients with intestinal complications were without immunosuppressive therapy at the time of our study, and nine out of thirteen - on immunosuppressive drugs. Routine laboratory and immunology testing could be beneficial for gastroenterologists in identifying patients at high risk for the development of complications and in the decision making for more aggressive therapy early after diagnosis.
\end{abstract}

Key words: Crohn's disease, complications, fecal calprotectin, fecal lactoferrin, immunosuppressive drugs

Corresponding author: Tsvetelina Velikova, MD, PhD, e-mail: ts_velikova@abv.bg 


\section{INTRODUCTION}

C rohn's disease $(C D)$ is a chronic inflammatory disorder which may involve every part of the gastrointestinal tract. The illness often passes asymptomatically and may take up to several years to be diagnosed [1]. CD may have some severe complications which negatively impact the quality of life and could lead to necessity of surgical treatment [2]. The complications in patients with $C D$ are two major types - intestinal and extraintestinal [3]. The most common intestinal complications are an obstruction, formation of a fistula, perforation, abscesses forming a collection of pus, and intestinal bleeding [4]. Patients are obliged to the chronic inflammation that produces scar tissue known as stricture and deep ulcers, which are very peculiar for $C D$ [1]. It is thought that immune and genetic factors (as HLA genes) are involved in the pathogenesis of extraintestinal manifestations.
Extraintestinal features in $C D$ patients are presented as eye complications, arthropathy, skin and oral lesions, delayed growth in children, etc. Some of these complications may have an association with disease activity, like episcleritis, but others, i.e., pyoderma gangrenosum, may not correlate with disease activity [3]. However, in general, extraintestinal manifestations tend to follow the clinical course of $C D$ and may have huge impact on the quality of life, morbidity and even mortality $[3,5,6]$. The complications that are associated with the activity of CD could respond to treatment of the bowel disease, but there is no specific treatment available for them [5]. Some conditions are associated with the damaged function of the intestine, like gallstones and kidney stones, anemia, osteomalacia, sensory disturbances (zinc deficiency, vitamin B deficiency) [1]. The delicate mechanism is related to the impaired absorption and malnutrition or the side effects of therapy (Figure 1) [7].

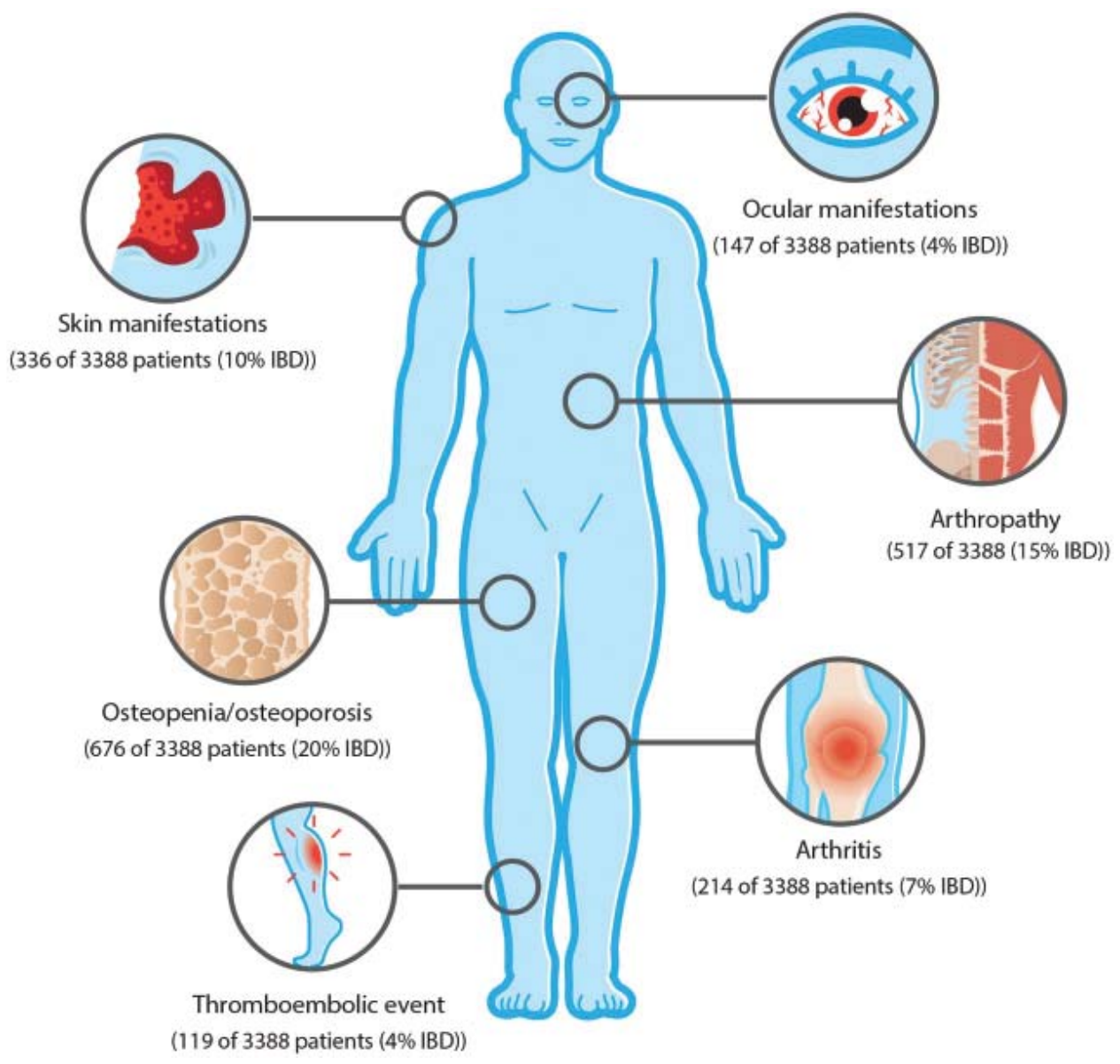

Fig. 1. The most frequent extraintestinal complications in Crohn's disease patients (Courtesy of Spekhorst, BMJ Open, 2017)

Both intestinal and extraintestinal complications contribute to increased morbidity and mortality of patients with CD. Thus, they are of growing health burden for these patients. This is the reason why it is crucial these complications and manifestations to be uncovered in time. The complications can occur, particularly during frequent or severe flare-ups [1]. Since there are some validated laboratory and immunologi- 
cal findings (i.e., CRP, ESR, fecal calprotectin, etc.) that may be used to assess the disease activity, we aimed to search for some associations between specific extraintestinal manifestation and intestinal complications in $C D$ patients with some clinico-laboratory findings and immunological markers. We also questioned whether the therapy administered influences the occurrence of complications.

\section{MATERIALS AND METHODS}

\section{Patients}

We examined retrospectively medical files of 26 patients with $C D$, diagnosed and treated in the Clinic of Gastroenterology at University Hospital "Sv. Ivan Rilski" from 2011 to 2012. The diagnosis was confirmed by colonoscopy plus biopsy and MRI.

All patients have signed the informed agreement, and the study was approved by the Ethic Committee of the Medical University of Sofia.

Eight men (30.8\%) and 18 women (69.2\%) at mean age $42 \pm 13$ years (range 21-71 years) were examined. The activity of the disease was assessed by CDAl (Crohn's disease activity index). The overwhelming proportion of the patients was at a state of activity $(77.9 \%)$. We divided patients into groups according to treatment regimens, Montreal classified localisation of the disease [8], and disease duration (Table 1). sum $(n=1)$, and intestinal complications: fistulas $(n=$ $6)$, obstructions $(n=2)$, anal fissures $(n=4)$. We also used the laboratory findings of the medical files for $\mathrm{C}$ reactive protein, iron, and hemoglobin in serum.

\section{Immunological methods}

The immunological markers fecal calprotectin (FC) and fecal lactoferrin (FL) were assessed in frozen $\left(-80^{\circ} \mathrm{C}\right)$ fecal samples of the patients by performing qualitative card-tests (One step card test, Certest, Spain) at the Laboratory of Clinical Immunology, University Hospital "Sv. Ivan Rilski", Sofia, according to manufacturer's instructions. The methods were based on lateral immunochromatographic diffusion, and the cut-off for both tests was $50 \mathrm{mg} / \mathrm{kg}$.

\section{Statistical methods}

We performed descriptive statistics, T-test, correlation and other analytical techniques to achieve the aim of the study by using SPSS v.19 (IBM). We accept the results as significant if $p<0.05$.

\section{RESULTS}

Overall, 19 (73\%) of the investigated CD patients had some extraintestinal manifestation and/or intestinal complications. We observed extraintestinal manifestations in 3/26 patients, $13 / 26$ were with intestinal complications, and $3 / 26$ had both (Table 2).

Table 1. Characteristics of the study subjects presented as number (\%)

\begin{tabular}{|l|l|l|}
\hline \multicolumn{2}{|l|}{ Characteristics of the patients } & Number (\%) \\
\hline \multirow{2}{*}{ Age } & Young patients (<44 years old) & $16(60 \%)$ \\
\cline { 2 - 3 } & Older patients (>44 years old) & $10(40 \%)$ \\
\hline \multirow{2}{*}{ Sex } & Male & $8(30.8 \%)$ \\
\cline { 2 - 3 } & Female & $18(69.2 \%)$ \\
\hline \multirow{2}{*}{ Disease activity } & CDAI < 150 (remission) & $6(23.1 \%)$ \\
\cline { 2 - 3 } & CDAl $>150$ (activity) & $20(77.9 \%)$ \\
\hline \multirow{3}{*}{$\begin{array}{l}\text { Localisation } \\
\text { Montreal classification) }\end{array}$} & Illeal - L1 & $2(8.7 \%)$ \\
\cline { 2 - 3 } & Colonic - L2 & $10(43.5 \%)$ \\
\cline { 2 - 3 } & Illeo-colonic - L3 & $11(47.8 \%)$ \\
\hline \multirow{5}{*}{ Duration of the disease } & None & $7(28 \%)$ \\
\cline { 2 - 3 } & Immunosuppression & $18(69.2 \%)$ \\
\cline { 2 - 3 } & Immunosuppression + Biologic & $1(4 \%)$ \\
\hline & $<1$ year & $7(28 \%)$ \\
\cline { 2 - 3 } & $1-10$ years & $15(60 \%)$ \\
\cline { 2 - 3 } & $>10$ years & $2(12 \%)$ \\
\hline
\end{tabular}

We collected clinical and laboratory data from medical files of the patients retrospectively. We found the following registered extraintestinal manifestations in patients: arthralgia/arthritis $(n=2)$, pyoderma gangreno-
Extraintestinal manifestations were documented more often in women with long-lasting and active disease (without reaching significance, $p>0.05$ ). All three patients with extraintestinal manifestations 


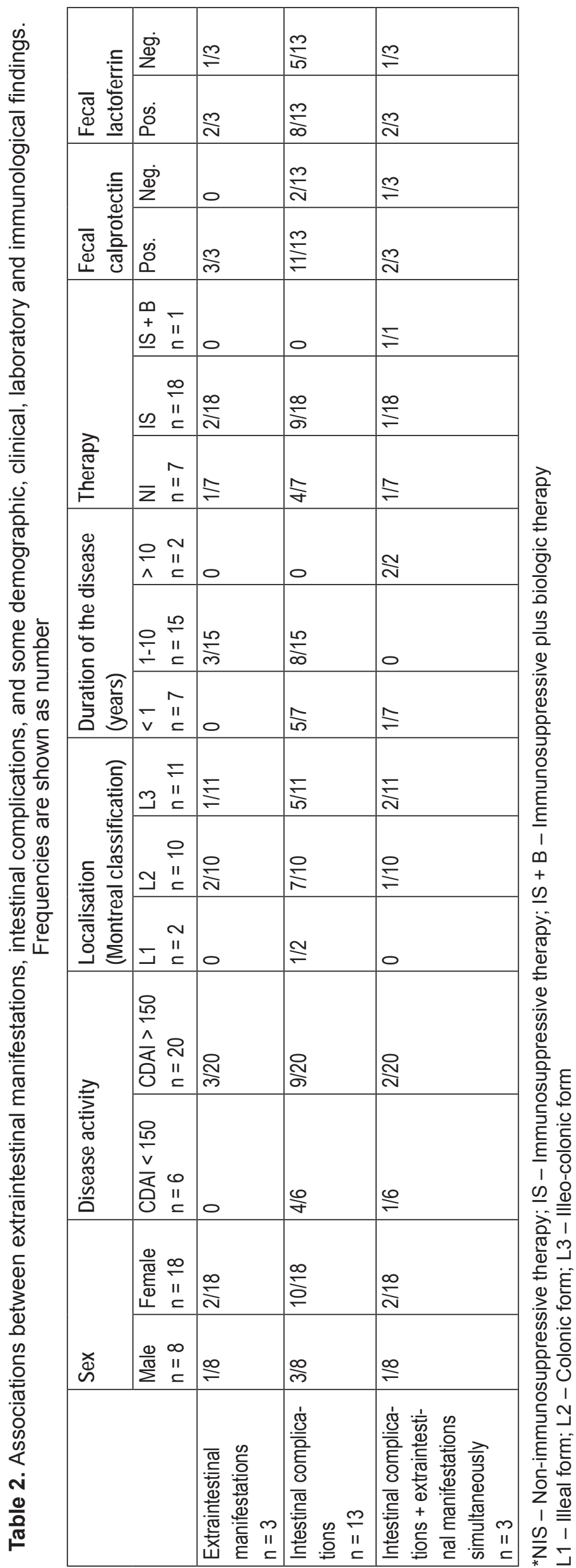

were positive for $\mathrm{FC}$ and $2 / 3$ were positive for FL (Table 2).

Intestinal complications occurred more often in patients with the active and long-lasting disease, however, non-significantly ( $p>0.05)$, also in women, in non-immunosuppressive treated patients, in patients with positive FC and FL $(p>0.05)$ (Table 2). The intestinal complications were more frequent in women than men $-55.6 \%$ vs. $37.5 \%$ respectively, regardless the state of activity $(66.7 \%$ of patients were at a state of remission, and $45.0 \%$ of patients were at a state of activity when they had complications). However, $37.5 \%$ of men had none complications during the disease.

The overwhelming proportion of patients $(66.7 \%)$ with long-lasting disease (up to 10 years) had at least one extraintestinal manifestation and/or intestinal complication, the most of these patients receiving non-immunosuppressive therapy, too.

The majority of the $C D$ patients with positive FC and FL had intestinal complications. Positive markers were associated with the active state of the disease (according to CDAl).

We also found differences in some laboratory markers depending on the presence of complications. We observed higher serum level of CRP (24.49 mg/l vs. $3.13 \mathrm{mg} / \mathrm{l}, \mathrm{p}=0.010$ ), slightly lowered serum level of hemoglobin $(120 \mathrm{~g} / \mathrm{l}$ vs. $145 \mathrm{~g} / \mathrm{l}, \mathrm{p}=0.044)$ and about 2-fold lower iron level $(7.23 \mu \mathrm{mol} / \mathrm{l}$ vs. $14.0 \mu \mathrm{mol} / \mathrm{l}, p=0.019)$ in patients with intestinal complications compared to patients without complications, respectively (Figure $2 \mathrm{~A}, \mathrm{~B}, \mathrm{C}$ ).

From the three patients with extraintestinal manifestations of the disease, one was on nonimmunosuppressive therapy and two - on immunosuppressive drugs. Four out of thirteen patients with intestinal complications were without immunosuppressive treatment at the time of our study, and nine out of thirteen - on immunosuppressive medications. Three patients with both intestinal and extraintestinal symptoms were divided in the three therapeutic groups, one in every group (non-immunosuppressive, immunosuppressive and immunosuppressive + biologic therapy) (Table 2). 

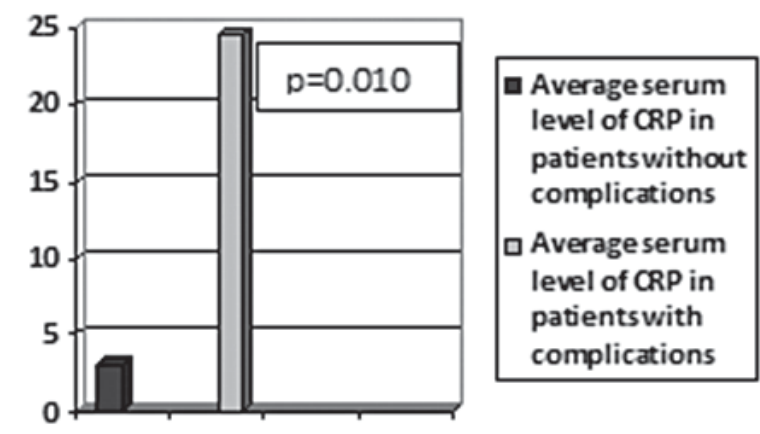

A.

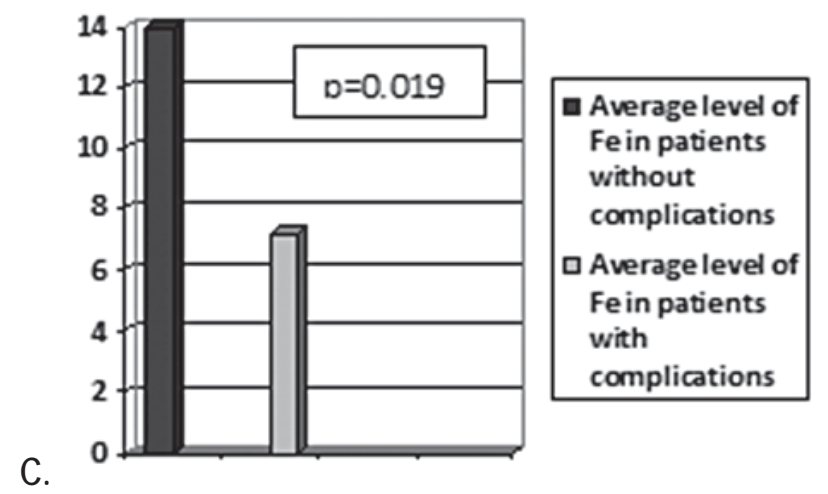

\section{DISCUSSION}

We found that patients with long-lasting (over ten years) disease have at least one intestinal complication/extraintestinal manifestation of $\mathrm{CD}(73 \%$ of the study group). This is not surprising since $C D$ is a chronic progressive disease that begins as an inflammation in the mucosa and then progressively develops over time to a more complex illness with stricture and fistula formation [9]. Furthermore, it is found that half of the patients with a lasting disease over 20 years suffered from intestinal complications and half of the hospitalized patients needed surgical treatment within ten years from diagnosis $[9,10]$. There are accumulated data that over $60 \%$ of $C D$ patients develop structuring and penetrating complications, where twenty-year actual rates of inflammatory, structuring and penetrating disease were $12 \%$, $18 \%$ and $70 \%$, respectively [11]. In our study $13 / 26$ (50\%) CD patients had intestinal complications which result is in line with the cited resources.

It is determined that disease phenotype is changed markedly over time, with nearly $80 \%$ of inflammatory disease ultimately evolving into structuring or penetrating pattern of behavior. [12]. Moreover, it is shown that $C D$ with early onset has more severe behavior

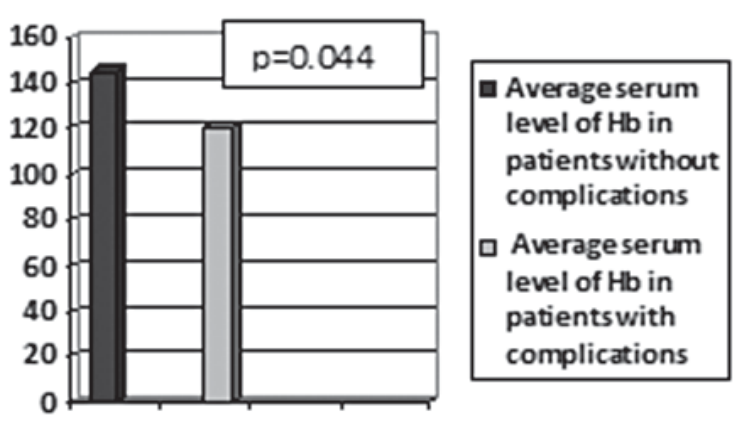

B.

Fig. 2. Some laboratory findings and the presence of complications in Crohn's disease patients: $\mathbf{A}$ - Serum $\mathbf{C}$-reactive protein, mg/l; B - Hemoglobin, g/l; C - Serum iron, $\mu \mathrm{mol} / \mathrm{l}$

and more extensive localization than the disease with a later start. It was mentioned above that the extraintestinal complications might be due to side effects of therapy or the damaged function of the intestines [12]. We found that our patients on immunosuppressive therapy had more intestinal complications than extraintestinal ones. The reason for that may lie in the fact that immunosuppressive drugs are necessary when the disease is severe which includes the presence of complications. Moreover, it is shown that early administration of immunosuppressants was associated with a decreased risk of surgical intervention for structuring and penetrating disease among CD patients [13-16]. The use of immunosuppressive medicaments has increased in the last 20 years, and it is not accidental. A multivariate Cox - regression analysis revealed that the time to initiation of Azathioprine therapy was significantly associated with the decade of $C D$ diagnosis $(p<0.001$, hazard ratio (HR) 2.88-6.53), age at onset $(p=0.008, H R$ 1.76), disease behavior at diagnosis $(p<0.001$, HR 1.762.07 ), need for systematic steroids ( $p<0.001, H R$ 2.71) and the time to intestinal surgery (HR 0.43 , [95\% Cl: 0.28-0.65]) [17]. According to extraintestinal manifestations/complications of $C D$, we observed such in about $15 \%$ of our cohort of patients. 
It is thought that some extraintestinal complications arise simultaneously during the flare of the bowel disease, and consequently, they can be influenced by the treatment of the flare.

The next aspect of our study was to investigate the association between complications and the levels of some common laboratory findings in $C D$ patients. CRP is documented to be increased during the active stage of the disease and may achieve up to and above $200 \mathrm{mg} / \mathrm{l}$ depending on the disease severity and the individual capacity of producing acute phase proteins. Furthermore, $86 \%$ of CD patients with elevated CRP exhibited inflammation at colonoscopy [18]. In our study, patients with intestinal complications showed about an 8-fold increase in CRP compared to patients without intestinal complications $(p=0.010)$. We also found alterations in hemoglobin $(p=0.044)$ and iron levels $(p=0.019)$ according to the presence of the complications. These tests are laboratory routine for $C D$ patients, including pediatric patients. Other common laboratory abnormalities in CD patients also enlist anemia, thrombocytosis (> $450 \times 109$ cells per $L$ ), and elevated ESR (> $20 \mathrm{~mm} /$ per hour). At least one abnormal result was found for $72 \%$ of patients with IBD, and more than one in about $49 \%$ of them [19].

We also found an association between high levels of fecal markers, FC and FL, and the frequency of complications in our group of CD patients. These outcomes are in confirmation of our previously published results [20]. Fecal markers are well accepted for diagnosis, prediction of activity and response to therapy. The sensitivity and specificity in identifying the activity of IBD by colonoscopy are shown to be $70-100 \%$ and $44-100 \%$, respectively $[18,21]$. In our study, we pointed out another utility of FC and FL, namely in identifying these patients with positive tests that may also have intestinal complications.

\section{CONCLUSION}

In our study, we obtained data in order to assess the associations between particular extraintestinal manifestation and intestinal complications in CD patients with some clinical and laboratory findings (CRP, hemoglobin, serum iron), immunological markers (fecal markers - FC and FL), and the administered therapy. These results could be beneficial for gastroenterologists in identifying patients at high risk for the development of complications and in the decision making for more aggressive therapy early after diagnosis.

Conflict of interests: None declared

Acknowledgement: None

\section{REFERENCES}

1. Friedman S et Blumberg S. Chapter 295 Inflammatory Bowel Disease. In: Harrison's Principles of Internal Medicine, 18 edition, Longo DL, Fauci AS, Kasper DL, Hauser SL, Jameson JL, Loscalzo J, USA, 2012.

2. Ha F et Khalil H. Crohn's disease: a clinical update. Ther Adv Gastroenterol, 2015, 8(6), 352-359.

3. Levine JS et Burakoff B. Extraintestinal Manifestations of Inflammatory Bowel Disease. Gastroenterology \& Hepatology, 2011, 7(4), 235-241.

4. Bernstein CN, Fried M, Krabshuis JH et al. World Gastroenterology Organization Practice Guidelines for the Diagnosis and Management of IBD in 2010. Inflammatory Bowel Diseases, 2010, 16(1), 112-114.

5. Rothfuss KS, Stange EF, Herrlinger KR. Extraintestinal manifestations and complications in inflammatory bowel diseases. World J Gastroenterol, 2006, 12(30), 4819-31.

6. Vavricka SR, Schoepfer A, Scharl M et al. Extraintestinal Manifestations of Inflammatory Bowel Disease. Inflamm Bowel Dis, 2015, 21, 1982-1992.

7. Spekhorst LM, Imhann F, Festen EA on behalf of the Parelsnoer Institute (PSI) and the Dutch Initiative on Crohn and Colitis (ICC), et al. Cohort profile: design and first results of the Dutch IBD Biobank: a prospective, nationwide biobank of patients with inflammatory bowel disease. BMJ Open, 2017, 7, e016695.

8. Satsangi J, Silverberg MS, Vermeire S et al. The Montreal classification of inflammatory bowel disease: controversies, consensus, and implications. Gut, 2006, 55, 749-753.

9. Freeman HJ. Natural history and long-term clinical course of Crohn's disease. World J Gastroenterol, 2014, 20(1), 31-36.

10. Hellers G. Crohn's disease in Stockholm county 1955-1974. A study of epidemiology, results of surgical treatment and longterm prognosis. Acta Chir Scand Suppl, 1979, 490, 1-84.

11. Cosnes J, Cattan S, Blain A et al. Long-term evolution of disease behavior of Crohn's disease. Inflamm Bowel Dis, 2002, 8(4), 244-50.

12. Fedorak RN. Is it time to re-classify Crohn's disease? Best Pract Res Clin Gastroenterol, 2004, 18 Suppl, 99-106.

13. Herfarth HH, Long MD, Kappelman MD. Immunosuppression for management of Crohn's disease. The Lancet, 2016, 387(10020), 747-748.

14. Khanna, R, Bressler, B, Levesque, BG et al. Early combined immunosuppression for the management of Crohn's disease (REACT): a cluster randomised controlled trial. Lancet, 2015, 386, 1825-1834.

15. D'Haens, G, Baert, F, van Assche, G et al. Early combined immunosuppression or conventional management in patients with newly diagnosed Crohn's disease: an open randomised trial. Lancet, 2008, 371, 660-667.

16. Colombel, JF, Sandborn, WJ, Reinisch, W et al. Infliximab, azathioprine, or combination therapy for Crohn's disease. N Engl J Med, 2010, 362, 1383-1395.

17. Lakatos PL, Lakatos L, Kiss LS et al. Treatment of extraintestinal manifestations in inflammatory bowel disease. Digestion, 2012, 86 (Suppl 1), 28-35.

18. Iskandar HN, Ciorba MA. Biomarkers in inflammatory bowel disease: current practices and recent advances. Transl Res, 2012, 159(4), 313-25.

19. Benor $S$, Russell GH, Silver $M$ et al. Shortcomings of the inflammatory bowel disease Serology 7 panel. Pediatrics, 2010, 125(6), 1230-6.

20. Velikova TV. Investigations of immunological parameters for intestinal inflammation in order to establish new markers for diagnosis and follow-up of inflammatory bowel disease. Dissertation 2014. Medical university of Sofia.

21. Lewis JD. The Utility of Biomarkers in the Diagnosis and Therapy of Inflammatory Bowel Disease. Gastroenterology, 2011, 140(6), 1817-1826. 